\title{
RESEARCH ON THE COVERAGE OF COLD-ROLLED FLAT PRODUCTS WITH FILM-FORMING MATERIALS
}

\author{
Simona BOICIUC \\ "Dunarea de Jos" University of Galati, Romania \\ e-mail: simonaboiciuc@yahoo.com
}

\begin{abstract}
The paper aims to characterize film-forming materials from the microstructural, mechanical point of view for the assessment of the elasticity and flexibility of films, and from the chemical point of view, to determine their resistance to various chemicals, distilled water, 3\% hydrochloric acid and 3\% sodium hydroxide and their behaviour at corrosion in saline mist. It aims to develop a coating technology with film-forming materials for protection against corrosion.
\end{abstract}

KEYWORDS: film-forming materials, anti-corrosion protection

\section{Introduction}

The film-forming materials, capable of forming films, are used in various industrial sectors, besides providing anti-corrosion protection and abrasion resistance, non-stick and anti-lubricant qualities, thermal or electrical insulation, high or low friction coefficient, decorative appearance [1].

In the case of use as protective materials, they are used in the form of solutions, emulsions or dispersions in different solvents, on the basis of which varnishes, paints, enamels, primers and grouts, plastisols, organosols are produced [1].

Varnishes are solutions of cellulose derivatives, natural or synthetic resins in volatile organic solvents (chlorinated hydrocarbons, benzene, lower alcohols, ethyl acetate or amyl) with or without the addition of vegetable oils or corrosion inhibitors. They are colourless or weakly coloured by the used resins or dyes. After drying they give transparent and glossy films. The dissolution of the film-forming material is carried out at a much lower temperature than the boiling temperature of the solvent [1].

Paints consist of a film-forming substance (natural or synthetic resins, cellulose and rubber derivatives, flax oil, bitumen), a suitable solvent, specific to the resin used (benzene, toluene, xylene alcohols, acetone) and a pigment (metal oxides, $\mathrm{Pb}_{3} \mathrm{O}_{4}, \mathrm{ZnO}, \mathrm{TiO}_{2}$, iron oxides, metals in finely dispersed state $\mathrm{Zn}, \mathrm{Al}$ ) in order to accelerate the polymerization of the film-forming substance or to provide film gloss [2].

To ensure corrosion protection the film must be sufficiently thick, chemically and mechanically resistant and stable against water soaking (electrolyte). The structure, porosity, permeability of the film depends on the nature of the resin, its concentration and the adhesion to the substrate.

The purpose of the paper is to establish a technology for coating flat products with filmforming materials (varnishes and paints). In this respect, a number of film-forming materials will be structurally, mechanically and chemically characterized.

\section{Experimental conditions}

\subsection{Sampling and coating with film- forming materials}

In the experimental researches, it was used a steel sheet, C10E type, with a thickness of $1 \mathrm{~mm}$.

The following technological flow was used to obtain the samples needed to coat with film-forming materials: mechanical pre-cleaning, alkaline chemical degreasing, hot washing, degreasing (tricolorethylene), washing, oxalic acid pickling, washing, phosphating, coating with film-forming materials (with brush or rolls), drying (polymerization).

The quality of the coating and its durability depend on the correct preparation of the surface before painting. In this case, surface cleaning was done mechanically with abrasive materials (fine abrasive polishing). The resulting roughness was 0.4 $0.6 \mu \mathrm{m}$.

The next step is the alkaline chemical degreasing which aims to remove fats (especially 
unsoapable fats). The solution used consists of $20 \mathrm{~g} / \mathrm{L}$ sodium hydroxide, $45 \mathrm{~g} / \mathrm{L}$ sodium carbonate, $20 \mathrm{~g} / \mathrm{L}$ trisodium phosphate, $3 \mathrm{~g} / \mathrm{L}$ surfactant (detergent). The working temperature was $70-75^{\circ} \mathrm{C}$, duration of $20-30$ seconds. After degreasing the samples were washed with demineralized water at a temperature of 60-65 ${ }^{\circ} \mathrm{C}$.

The next step consists of degreasing in tricolorethylene followed by washing and pickling in $20 \mathrm{~g} / \mathrm{L}$ oxalic acid at a temperature of $18-25^{\circ} \mathrm{C}$. After degreasing the samples were washed with demineralized water at room temperature.

Phosphating aims to form on the metal surface a layer of fine crystals of insoluble secondary and tertiary metal phosphates (iron, magnesium or zinc phosphates) from aqueous solutions containing primary metal phosphates. The solution heated to temperatures ranging from $40-100{ }^{\circ} \mathrm{C}$, upon immersion of the sample, attacks its surface due to the acid present, and a small amount of metal passes into the solution together with the formation of a phosphate deposit. In this process, the surface of the metal (sample) is presented as a polyelectrode which implies the existence of micropiles composed of anodic areas (where the metal dissolves) and cathodic areas (where hydrogen is released) in the acid solution [3].

The phosphate film provides anti-corrosion protection in combination with other film-forming materials (service life 5 to 10 years). Their deposition is favoured by the intercrystalline porosity $(0.1-5 \%)$, the resulting roughness $(0.7-0.9 \mu \mathrm{m})$ of the phosphate layer and their absorbing properties. The layer obtained has good stability, is hard, and withstands temperatures up to approx. $200{ }^{\circ} \mathrm{C}$. It can also be useful for increasing wear resistance, as a solid lubricant and a lubricant in the cold deformation process (cleavage planes appear that facilitate the movement of crystalline particles during cold plastic deformation) [3].

The phosphating solution used in the experimental research has the following composition: phosphoric acid $418 \mathrm{~g} / \mathrm{L}$, nitric acid $130 \mathrm{~g} / \mathrm{L}, \mathrm{Zn} 162$ $\mathrm{g} / \mathrm{L}$, Ni $0.65 \mathrm{~g} / \mathrm{L}$. The samples were immersed in the solution at a temperature of $80-90{ }^{\circ} \mathrm{C}$ where they were kept approx. 3-4 minutes (until the beginning of the hydrogen release).

After the operations presented above, the samples were subjected to coating (with a brush) with film-forming materials in a number of 4 layers. The number of layers is determined according to the thickness of the film and the nature of the materials used (the thickness of an individual layer is about 20$30 \mu \mathrm{m})$. After coating and drying the samples were placed in the oven at temperatures of approx. 100-160 ${ }^{\circ} \mathrm{C}$ (depending on the type of coating) for further polymerization (increasing the mechanical strength of the film).

The coatings were made with the following types of film-forming materials.

1. Alorex (P1) - black colour, is a paint composed of two components: the epoxy component and the hardener (mix in a ratio of 5:1). The crosslinking time of the polymer is 24 hours. They are thermoreactive products obtained by condensing dioxifenylpropane with epichlorohydrin. They have a greater flexibility (greater than the phenolic ones) and a better chemical resistance than the alkyd resins. They are resistant to most inorganic salts, their solutions, to oxidizing ones, to the action of bases and of aliphatic alcohols as well as of hydrocarbons.

These resins can form films only as a result of the reaction of polyaddition with polyaminoamides, anhydrides, polyisocyanates, by oven drying in combination with resins containing hydroxyl groups.

2. Email epoxidic seria $3100(\mathrm{P} 2)$ - red colour, is made from high molecular weight epoxy resins that form films by cross-linking with polyamide adducts. The film is formed by chemical conversion in combination with 3304 epoxy-polyamide hardeners at room temperature or in the oven. Drying is carried out at temperatures between $80-120{ }^{\circ} \mathrm{C}$. It provides colorful, smooth, glossy, hard films, with high coverage power, resistant to industrial atmosphere.

3. Rodin (P3) - transparent film, is a product made of nestrapol and a hardener (based on epoxy resins). For the preparation, mix $100 \mathrm{~g}$ nestrapol with $35 \mathrm{~g}$ of hardener and stir for 15 minutes. The mixture obtained lasts 30 to 50 minutes depending on the ambient temperature (this duration decreases with increasing temperature). The applied layer dries at temperatures of $80-100{ }^{\circ} \mathrm{C}$. Nestrapol (450) is an unsaturated, orthophthalic polyester resin. It is delivered as a solution $66 \%$ in styrene. Cobalt naphthenate entering the preparation of nestrapol is a product obtained by saponification of organic acids and precipitation with cobalt nitrate solution. The product is flammable (temperature $28^{\circ} \mathrm{C}$ ) and toxic. Esterified epoxy paints have good weather resistance, are easy to apply but lose their shine over time, are expensive and dry slowly.

The samples thus obtained were characterized from micro-structural, mechanical point of view for the assessment of the elasticity and flexibility of the films and chemically, for determining their resistance to different chemicals as well as the corrosion behaviour in saline mist. 


\subsection{Characterization of the films obtained}

\subsubsection{Microscopic characterization of films}

The microstructure of the obtained film-forming materials was revealed by optical microscopy using a Neophot 2 microscope.

\subsubsection{Determining the elasticity of films}

The elasticity of the film was determined by stamping the metal support on which the film material is applied. After the test the film must not show cracks or exfoliation. The test was performed using a spherical punch with a diameter of $20 \mathrm{~mm}, \mathrm{~F}$ $=10 \mathrm{kN}$. The samples had the dimensions of $210 \times 70$ $\mathrm{mm}$.

\subsubsection{Determining the flexibility of films}

The flexibility of the film was determined by bending at $180^{\circ}$ of the substrate on which it is applied, on cylindrical spindles with dimensions of $\phi 32 \mathrm{~mm}$ and $\phi 0.3 \mathrm{~mm}$ under certain conditions and observing its behaviour. After the test the film must not show cracks or exfoliation.

\subsubsection{Determining the resistance of films to liquids}

The resistance of films to the action of some liquids is their ability not to modify their initial properties under the action of the respective liquid. To determine this, the samples $(100 \times 500 \mathrm{~mm})$ coated with the film-forming materials were immersed in distilled water, $3 \%$ hydrochloric acid and $3 \%$ sodium hydroxide, at a temperature of $23 \pm 2{ }^{\circ} \mathrm{C}$ for 24 hours. After the test the film must not show cracks, exfoliation or swelling.

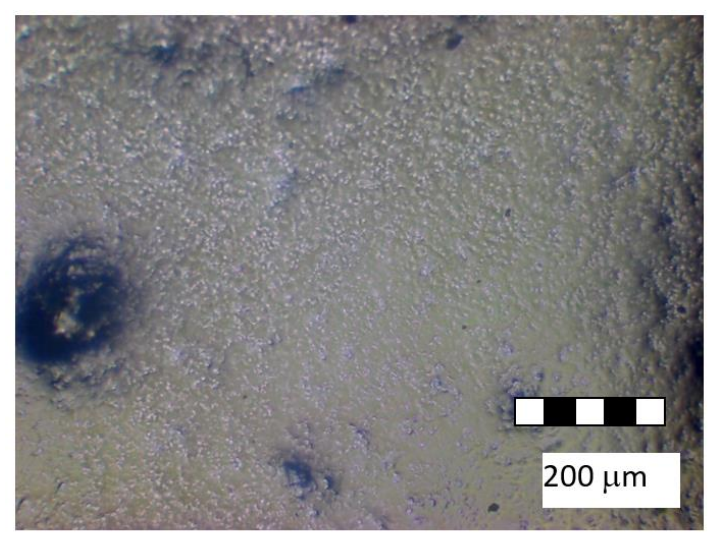

P1

\subsubsection{Corrosion behaviour of films}

The corrosion behaviour in saline mist of the film-forming materials was studied using a corrosion chamber, provided with an automatic system for measuring and regulating the temperature, exhaust system of the used solution or of the condensation, resulted during the tests.

The samples were placed on plastic bars.

The accelerated corrosion test in saline mist was performed according to SRISO 9227.

To perform the test, the necessary solution was prepared, by dissolving in distilled water, with a conductivity less than or equal to $20 \mu \mathrm{S} / \mathrm{cm}^{2}$ at $35 \pm 2$ ${ }^{\circ} \mathrm{C}$, of a quantity of sodium chloride, to obtain a concentration of $50 \pm 5 \mathrm{~g} / \mathrm{L}$. The relative density of a solution with this concentration ranges from 1.00255 to 1.0400 .

The $\mathrm{pH}$ of the salt solution was adjusted so that the $\mathrm{pH}$ of the solution collected in the spray chamber is between 6.5 and 7.2. It was measured with a pH meter HI 991001, produced by "Hanna Instruments", provided with a temperature indicator.

The corrosion test was performed for a period of 120 hours at $35{ }^{\circ} \mathrm{C}$. Initially the samples were degreased with alcohol, weighed on the analytical balance with an accuracy of $0.01 \mathrm{mg}$.

At an interval of 24 hours, the samples were removed from the chamber, the corrosion products were removed by washing with water and then dried. Subsequently, the weighing on the analytical balance was carried out with an accuracy of $0.01 \mathrm{mg}$.

\section{Experimental results}

\subsection{Microscopic characterization of the obtained films}

Following the microscopic analysis, it was observed that the obtained films, show no cracks, are homogeneous and adherent as can be seen in Figure 1.

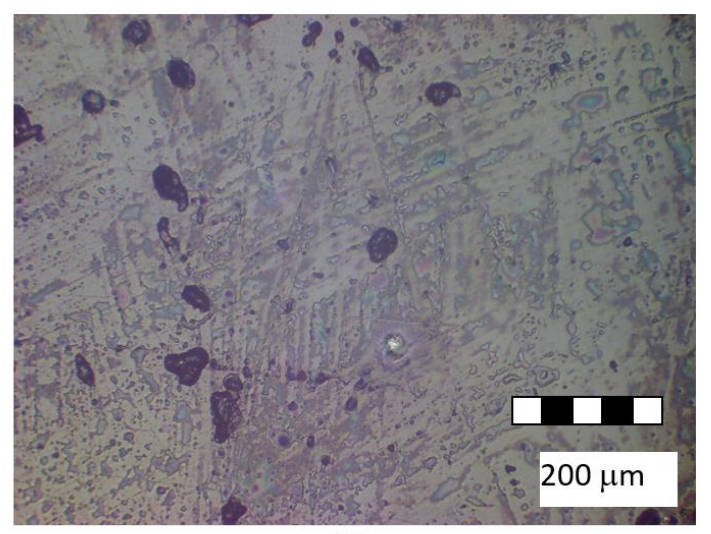

P2 


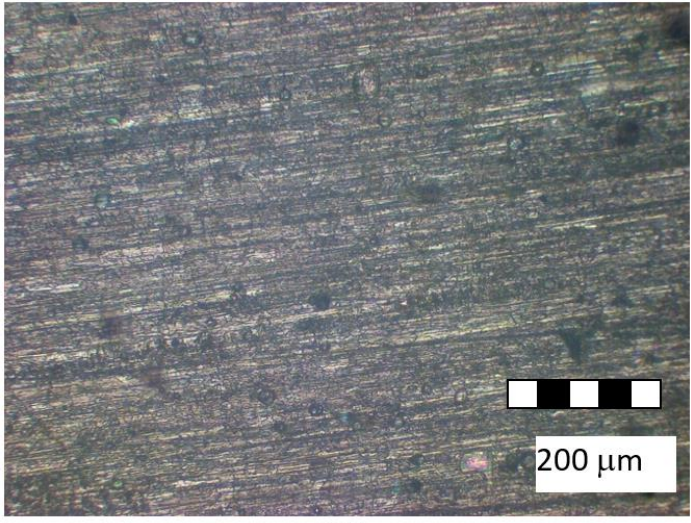

P3

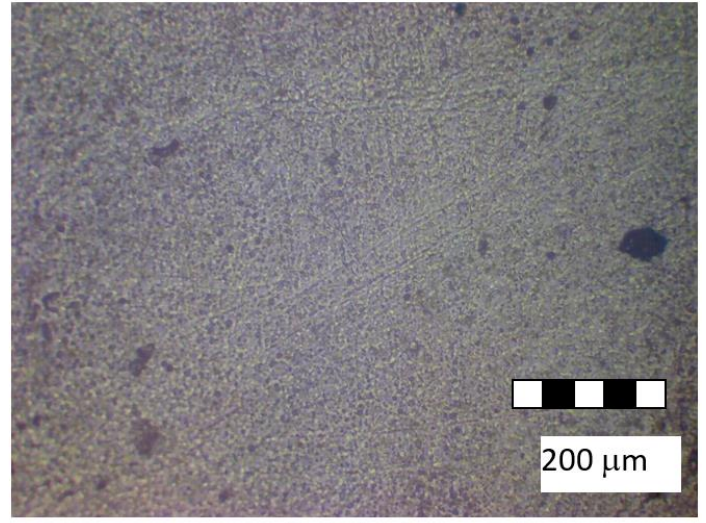

Substrate

Fig. 1. The metallographic aspect of the film-forming materials

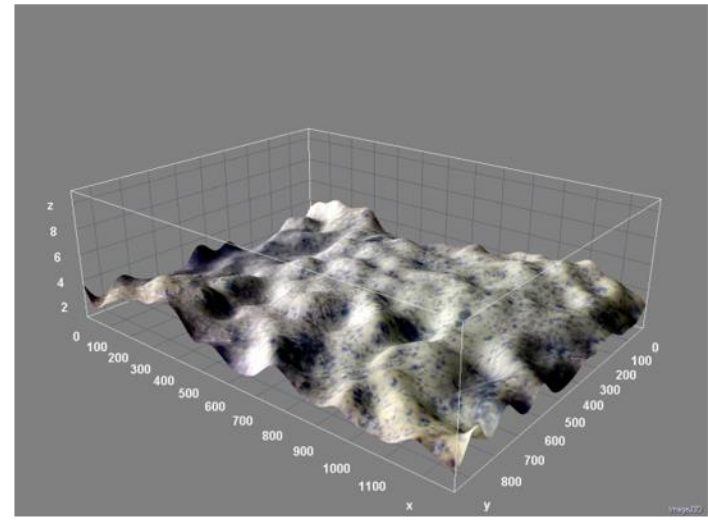

P1

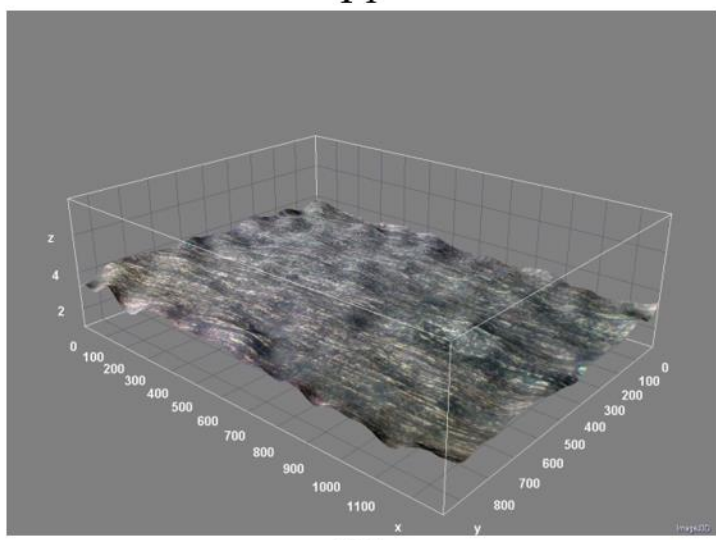

P3

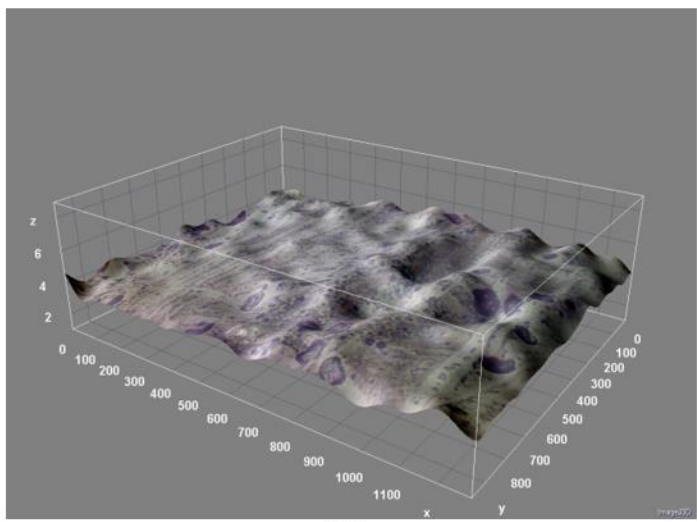

P2

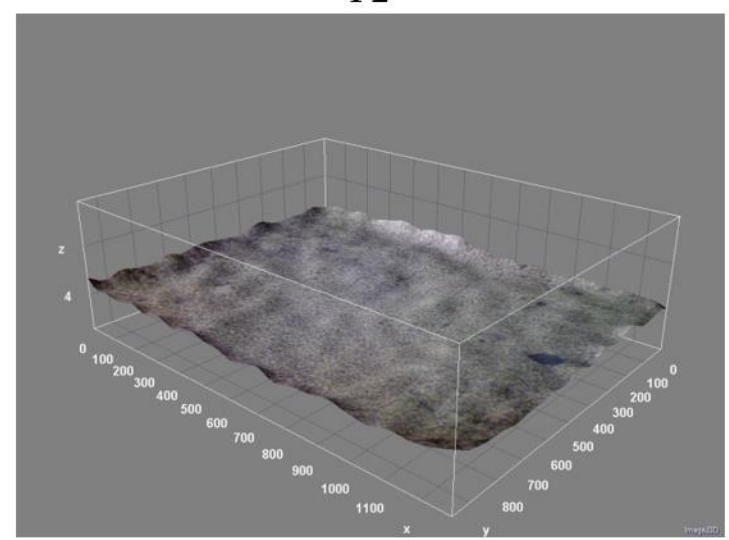

Substrate

Fig. 2. 3D images of film-forming materials

Fig. 2 shows the qualitative, 3D aspect, of the surface profile for the obtained film-forming materials. It can be seen that the film P3 - Rodin shows a higher uniformity (lower roughness) compared to the films P1 - Alorex and P2 - Epoxy enamel 3100 series.

\subsection{Determining the elasticity of films}

Analysing Table 1 it can be observed that after stamping the substrate on which the film-forming material is applied, it was found that the best elasticity is noticed to sample P3 - Rodin and the lowest to sample P1 - Alorex. 
Table 1. Determining the elasticity of films

\begin{tabular}{|c|c|c|c|c|}
\hline \multirow{2}{*}{$\begin{array}{c}\text { Erichsen index } \\
\mathrm{I}_{\mathrm{E}}[\mathrm{mm}]\end{array}$} & P1 - Alorex & $\begin{array}{c}\text { P2 - Epoxy enamel } \\
\text { 3100 series }\end{array}$ & P3 - Rodin & Substrate \\
\cline { 2 - 5 } & 1.4 & 1.6 & 8.8 & 11 \\
\hline
\end{tabular}

\subsection{Determining the flexibility of films}

After bending the samples at $180^{\circ}$ on cylindrical spindles with dimensions of $\phi 32 \mathrm{~mm}$ and $\phi 0.3 \mathrm{~mm}$ it was noticed that the best flexibility was found to the P3 - Rodin sample. The other films showed cracks and exfoliation even when bent on the large spindle.

\subsection{Determining the resistance of films to liquids}

After immersion of the samples, 24 hours, in distilled water, $3 \%$ hydrochloric acid and $3 \%$ sodium hydroxide, it was found that they withstood well in distilled water and 3\% sodium hydroxide, and of those immersed in 3\% hydrochloric acid only those covered with Epoxy enamel 3100 series showed swelling and exfoliation.

The anticorrosive protection of film-forming materials is a complex phenomenon because they have pores and capillaries which makes them permeable to liquids. Thus, their most important role is to reduce the speed of the electrochemical reactions of the corrosion process, by making it difficult for the film to access the metal surface of the depolarized elements and ions $[1,2]$.

When the paint film is in a certain environment, it is subjected to absorption phenomena and diffusion forces that allow it to penetrate to the surface of the substrate. The film thus becomes the site of osmotic phenomena due to its porous structure. The electrolyte absorption can usually be achieved by electroosmosis (due to potential differences) or by osmosis (due to differences in ion concentration) [1, 2].

Thus, it is necessary for the paint film (usually negatively charged) to have a capillary system that has a negative charge of the walls against the electrolyte solutions because in this way the electroosmotic dehydration of the anodic zones takes place, not allowing the substrate to dissolve. The electroosmotic flow of water or electrolytes occurs from anodic to cathodic areas (formed on the surface of the substrate) $[1,2]$.

On the other hand, the capillary system must be sufficiently permeable to facilitate the removal of liquid from the cathodic areas and release of the discharged hydrogen into these areas. Otherwise, swelling occurs in these areas [1,2].

In these conditions, it is important to achieve the passivation the substrate favoured by the phosphating performed prior to the deposition of the film-forming material.

\subsection{Corrosion behaviour of films}

Analysing Fig. 3 it can be seen that the best corrosion behaviour in saline mist is noticed on sample P1 - Alorex and the lowest on sample P3 Rodin with a maximum at 24 hours. This behaviour is due to the lower absorption capacity of the electrolyte solution by sample P1.

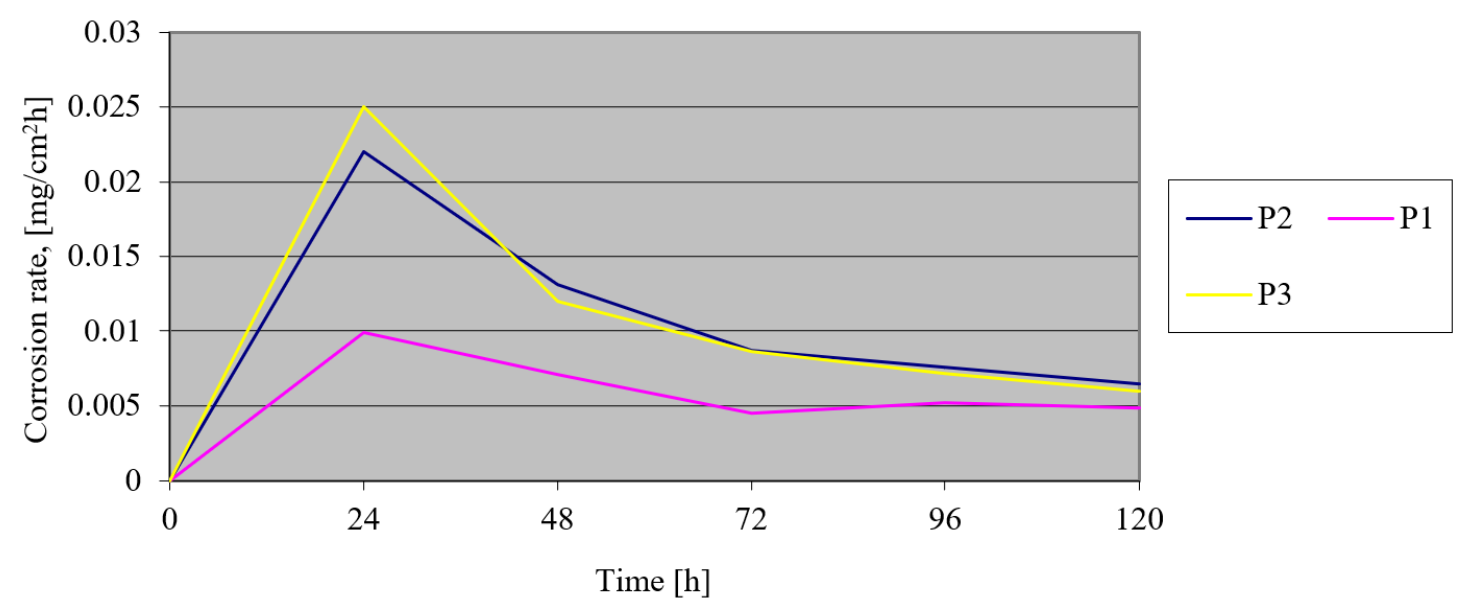

Fig. 3. Corrosion behaviour in saline mist of film-forming materials 


\section{Conclusions}

Following the testing of the film-forming materials applied on steel substrate results in the following conclusions:

- the films obtained have no cracks, are homogeneous and adherent;

- the film elasticity test performed by stamping the metallic substrate showed that the Rodin film is the most elastic one IE $=8.8 \mathrm{~mm}$ and the most rigid one is the Alorex film IE $=1.4 \mathrm{~mm}$ (IE substrate $=11 \mathrm{~mm}$ );

- the best flexibility was noticed on Rodin film, the other films showing cracks and exfoliation after bending the substrate to $180 \square$ on cylindrical spindles with different diameters; this behaviour is due to the presence in the composition of nestrapol;

- following the test to determine the resistance of films to liquids it was found that the samples have a good resistance in distilled water and $3 \% \mathrm{NaOH}$ and of those immersed in $3 \% \mathrm{HCl}$ only those with Email 3100 showed swelling and exfoliation on the surface of the substrate;
- the corrosion test performed in saline mist showed that the best behaviour was noticed on sample P1 - Alorex and the lowest on sample P3 Rodin with a maximum at 24 hours;

- to ensure anticorrosive protection, the filmforming material should be soaked with water, electrolyte solutions, have a negative charge against them, have a reduced capillary system to oppose a higher ohmic resistance, thus ensuring a low corrosion rate; on the other hand, it must be sufficiently loose to allow the electroosmotic water to flow outwards.

\section{References}

[1]. Maria Constantinescu, Protecţia anticorozivă a metalelor, Editura Tehnică, Bucureşti, 1979.

[2]. Ioan Rădoi, Introducere în coroziunea şi protecţia metalelor şi aliajelor, Editura Facla, 1982

[3]. Marinescu A., Andonianț Gh., Bay E., Tehnologii electrochimice şi chimice de protectie a materialelor metalice, Editura Tehnica București, 1984.

[4]. Alexandru Constantinescu, Detectarea şi măsurarea coroziunii, Editura Tehnică, Bucureşti, 1976. 